\title{
Inefficiencies in unbalanced three-phase power systems. Relationship between system asymmetry and instantaneous power waves
}

\author{
R. Sabater, V. Donderis \\ Department of Electrical Engineering \\ Universitat Politècnica de Valencia \\ Camí de Vera, 14, 46022 València \\ phone:+34 9638775 98, fax:+34 9638775 99, e-mail: rsabater@die.upv.es, vdonderis@die.upv.es
}

\begin{abstract}
This work analyzes the energy phenomena occurring in linear power systems characterized by the presence of load unbalances, using the principles of the Unifying General Theory of Electric Power [1],[2].
\end{abstract}

The energy fluxes characterize each phenomenon in the power system, especially the energy associated with the asymmetry phenomenon; thus it is possible to quantify the asymmetry phenomenon from the instantaneous power shapes, manifested by three unbalanced sinusoidal fluxes, whose resultant is a sinusoidal wave.

In addition, this work presents the relationship between the quantification of the phenomenon and the amplitudes of the power fluxes.

\section{Key words}

Instantaneous power, load unbalances, inefficiencies, power quality modeling, energy transfer.

\section{Introduction}

Energy phenomena in three-phase power systems have been analyzed as a generalization of the phenomena occurring in single-phase circuits. Thus, in linear singlephase power systems we can distinguish the energy phenomena associated with the net energy transfer and with the dephase phenomenon, quantified by the active power $(\mathrm{P})$ and the reactive power $(\mathrm{Q})$.

The analysis of three-phase power systems using a similar approach to that used for single-phase power systems can lead to an improper identification and quantification of the energy phenomena, since if unbalances occur in the system, then in addition to the energy phenomena mentioned above, the phenomenon of asymmetry can also take place; this latter phenomenon does not happen in single-phase power systems and it should be quantified independently of the phenomena of useful energy transfer and dephase, differentiating and quantifying the effects of each of the energy phenomena occurring in the system.

In this paper we analyze the energy phenomena occurring in unbalanced three-phase linear power systems, using the Unifying General Theory of Electric Power [1],[2]. Each energy phenomenon in the power system is identified from the expression of instantaneous power and is quantified using the likelihood criterion [1],[2]. In addition, it is possible to quantify the different energy phenomena from their representations as a time function of the instantaneous power, emphasizing the relationship between the shape of the instantaneous asymmetry power and the quantification of the associated phenomenon.

\section{Expressions of instantaneous power in load-unbalanced three-phase linear power systems}

The instantaneous power in a three-phase power system with efficient generators and unbalanced loads connected in triangle can be expressed using Stokvis theorem (considering the excitations from direct sequence):

$$
p(t)=\sum_{j=R S, S T, T R} v_{j d}(t) \cdot i_{j d}(t)+\sum_{j=R S, S T, T R} v_{j d}(t)\left[i_{j i}(t)+i_{j h}(t)\right]
$$

where the first term is the product of the direct voltage and current components, and the second term corresponds to the inverse and homopolar components of the current.

From expression (1) and decomposing the direct current into its active and reactive components, we obtain the terms that characterize the phenomena established in Steinmetz theory: a one-directional flux of useful energy and a two-directional flux associated with the dephase phenomenon (2). 


$$
\begin{gathered}
\sum_{j=R S, S T, T R} v_{j d}(t) \cdot i_{j d}(t)= \\
=\sum_{j=R S, S T, T R} v_{j d}(t) \cdot i_{j d a}(t)+\sum_{j=R S, S T, T R} v_{j m}(t) \cdot i_{j d r}(t)= \\
=p_{p}(t)+p_{q}(t)
\end{gathered}
$$

The second term in expression (1) is not associated with any of the previous phenomena; therefore it is necessary to employ an electric power approach that explains the energy phenomena occurring in the system.

The Unifying General Theory of Electric Power defines the effective instantaneous power $p_{p}(t)$, as a onedirectional flux of load-generator power, associated with the direct voltage component (3), and the instantaneous dephase power $\mathrm{p}_{\mathrm{q}}(\mathrm{t})$ as a two-directional flux between generator and receiver associated with the product of the direct voltage and the reactive component of direct current (3):

$$
\begin{aligned}
& p_{p}(t)=\sum_{j=R S, S T, T R} v_{j d}(t) \cdot i_{j d a}(t) \\
& p_{q}(t)=\sum_{j=R S, S T, T R} v_{j m}(t) \cdot i_{j d r}(t)
\end{aligned}
$$

The effective power and dephase power are energy fluxes that characterize balanced three-phase linear power systems. When unbalances occur in the power system, then the second term of the expression for instantaneous power (1) occurs. It defines the instantaneous asymmetry power $\mathrm{p}_{\mathrm{A}}(\mathrm{t})$, associated with the inverse and homopolar components of the current (4):

$$
\begin{gathered}
p_{A}(t)=\sum_{j=R S, S T, T R}\left(v_{j d}(t) \cdot\left(i_{j i}(t)+i_{j h}(t)\right)\right)= \\
=\sum_{\substack{j=R S, S T, T R \\
k=i, h}} v_{j d}(t) \cdot i_{j k}(t)
\end{gathered}
$$

\section{Quantification of the energy phenomena}

The quantification of the energy phenomena is obtained using the likelihood criterion [1],[2] which provides the expressions that allow to obtain the effective power $\left(\mathrm{P}_{\mathrm{u}}\right)$, the dephase power $\left(Q_{u}\right)$, and the asymmetry power $\left(A_{u}\right)$.

$$
\begin{aligned}
& P_{u}=3 V_{d} \cdot I_{d a}=3 V_{d} \cdot I_{d} \cdot \cos \varphi_{d} \\
& Q_{u}=3 V_{d} \cdot I_{d r}=3 V_{d} \cdot I_{d} \cdot \operatorname{sen} \varphi_{d} \\
& A_{u}=3 V_{d} \sqrt{\left(I_{i}^{2}+I_{h}^{2}\right)}
\end{aligned}
$$

The apparent power $\left(\mathrm{S}_{\mathrm{u}}\right)$ coincides with Buccholz expression $\left(\mathrm{S}_{\mathrm{B}}\right)$ :

$$
\begin{aligned}
& S_{u}=\sqrt{P_{u}^{2}+Q_{u}^{2}+A_{u}^{2}}=3 V_{d} \sqrt{I_{d}^{2}+I_{i}^{2}+I_{h}^{2}} \\
& S_{B}=\sqrt{\left(V_{R S}^{2}+V_{S T}^{2}+V_{T R}^{2}\right) \cdot\left(I_{R S}^{2}+I_{S T}^{2}+I_{T R}^{2}\right)}
\end{aligned}
$$

Where $V_{d}, I_{d}, I_{i}$, and $I_{h}$ are the RMS values of the symmetrical components (phase values) of the system and $\mathrm{V}_{\mathrm{RS}}, \mathrm{V}_{\mathrm{ST}}, \mathrm{V}_{\mathrm{TR}}, \mathrm{I}_{\mathrm{RS}}, \mathrm{I}_{\mathrm{ST}}$ and $\mathrm{I}_{\mathrm{TR}}$ are the RMS values of the voltages and currents indicated.

\section{Effective power and dephase power}

From the expressions of the instantaneous power it is possible to obtain the wave shapes that represent each of the energy phenomena present in the power systems under analysis.

\section{A. Instantaneous effective power}

The instantaneous effective power $\mathrm{p}_{\mathrm{p}}(\mathrm{t})$ is characterized by being a senoid for each phase, whose resultant is a one-directional flux of load-generator (Fig.1).

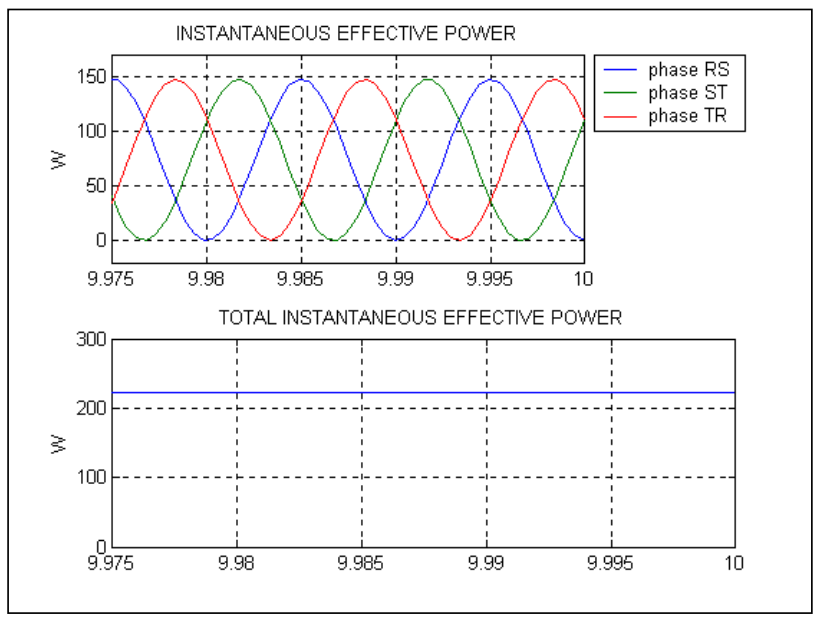

Fig.1. Instantaneous Effective Power

The quantification of the effective power can be obtained as the sum of the instantaneous values of the effective powers of each phase, the resultant being a onedirectional net power flux of value $P_{u}$.

$$
P_{u}=p_{\mathrm{RS} p}(t)+p_{\mathrm{ST} p}(t)+p_{\mathrm{TR} p}(t)=\sum_{j=R S, S T, T R} p_{j p}(t)
$$

\section{B. Instantaneous Dephase power}

Instantaneous dephase power $\mathrm{p}_{\mathrm{q}}(\mathrm{t})$ corresponds to a balanced sinusoidal three-phase flux (Fig. 2), of nil mean value (8):

$$
Q_{u}=p_{\mathrm{RS} q}(t)+p_{\mathrm{ST} q}(t)+p_{\mathrm{TR} q}(t)=0
$$




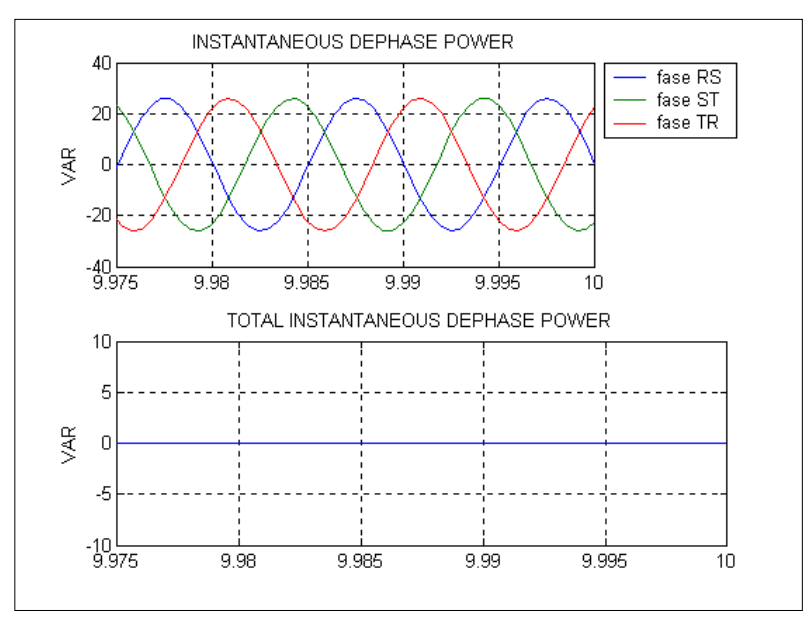

Fig. 2. Instantaneous Dephase Power

The quantification of the dephase power is obtained as the sum of the instantaneous power amplitudes of each phase:

$$
\begin{gathered}
Q_{u}=\max \left[p_{\mathrm{RS} q}(t)\right]+\max \left[p_{\mathrm{ST} q}(t)\right]+\max \left[p_{\mathrm{TR} q}(t)\right]= \\
=\sum_{j=R S, S T, T R} \max \left[p_{j q}(t)\right]
\end{gathered}
$$

\section{Instantaneous asymmetry power. Relationship between wave shape and the quantification of the energy phenomenon}

The instantaneous asymmetry power has been represented in Fig. 3, forming an unbalanced three-phase power system, whose resultant is a two-directional flux between generator and load of nil mean value. Initially there is no evident relationship between wave shape and the quantification of the phenomenon.

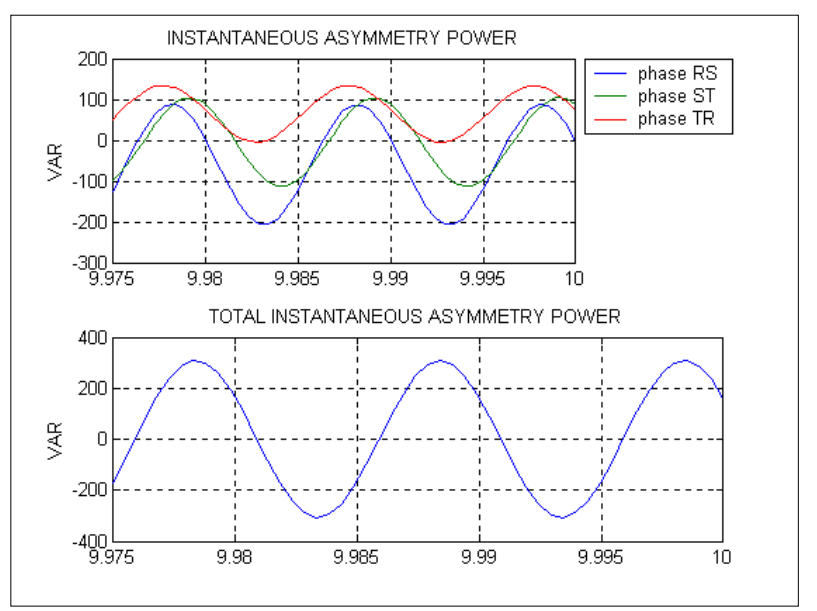

Fig. 3. Instantaneous Asymmetry Power
However, from the decomposition of the asymmetry power into its components associated with phase inverse current and phase homopolar current we can obtain the relationship between the energy phenomenon and its quantification.

A. Instantaneous asymmetry power associated with the inverse component of current

$$
p_{A i}(t)=\sum_{\substack{j=R, S T, T R \\ k=i}} v_{j d}(t) \cdot i_{j k}(t)
$$

The instantaneous power associated with the inverse component of the current (10) has been represented in Fig 4; it is formed by an unbalanced three-phase power system whose resultant is a senoid of nil mean value.

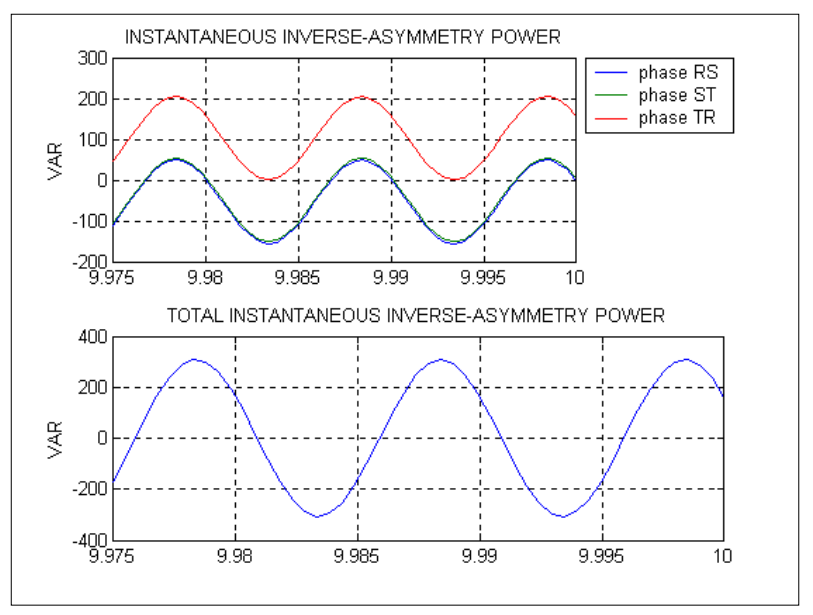

Fig. 4. Instantaneous Inverse-Asymmetry Power

The asymmetry power associated with the inverse component of the current can be obtained from the maximum value that represents the resultant of the powers of each phase, a result that coincides with the sum of the maximum values of the instantaneous asymmetry power associated with the inverse component of the current (11).

$$
A_{\text {inverse }}=\sum_{j=R S, S T, T R} \max \left[p_{j A i}(t)\right]
$$

B. Instantaneous asymmetry power associated with the homopolar component of the current

$$
p_{A h}(t)=\sum_{\substack{j=R S, S T, T R \\ k=h}} v_{j d}(t) \cdot i_{j k}(t)
$$

The instantaneous power associated with the homopolar component of the current (12) has been represented in Fig 5; it is formed by an unbalanced three-phase power system whose total sum phase by phase is nil. 


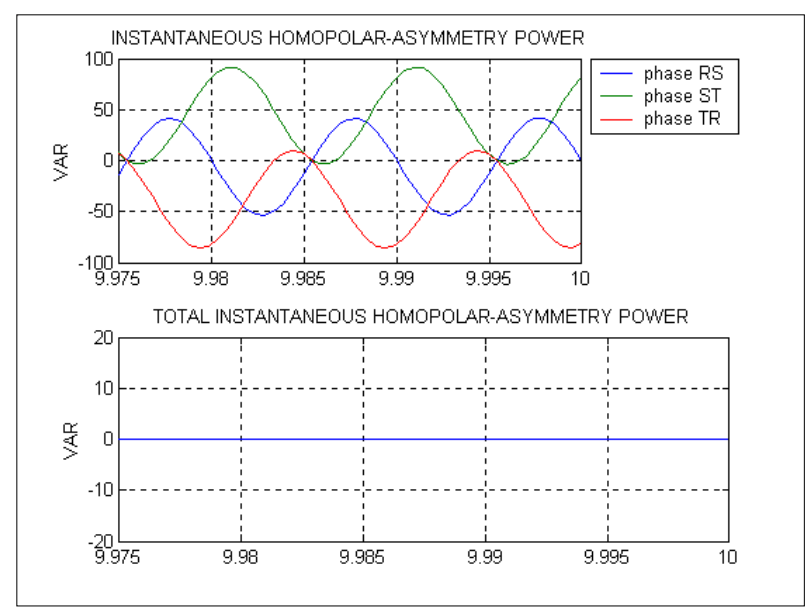

Fig. 5. Instantaneous Homopolar-Asymmetry Power

The quantification of the asymmetry power associated with the homopolar component of the current can be obtained from the sum of the maximum values of the instantaneous power of each phase (13):

$$
A_{\text {homopolar }}=\sum_{j=R S, S T, T R} \max \left[p_{j A h}(t)\right]
$$

\section{Asymmetry power. Quantification}

The relationship between the quantification of the asymmetry power and the wave shapes of its components is obtained as the quadratic sum of the asymmetry powers associated with the inverse and homopolar components of the phase current (14):

$$
\begin{gathered}
A_{u}=\sqrt{\left[\sum_{\substack{j=\\
R S, S T, T R}} \max \left[p_{j A i}(t)\right]\right]^{2}+\left[\sum_{\substack{j=\\
R S, S T, T R}} \max \left[p_{j A h}(t)\right]\right]^{2}}= \\
=\sqrt{A_{i}^{2}+A_{h}^{2}}
\end{gathered}
$$

The expression indicated in (14) allows us to calculate the value of the asymmetry power from the wave shapes of the instantaneous power components, allowing to obtain the relationship between the energy phenomenon and its quantification.

\section{Practical Case}

In order to verify the issues presented in previous paragraphs, we present a practical case of a three-phase linear power system with unbalanced loads connected in triangle with the following data:

$$
\begin{array}{ll}
\bar{V}_{R S}=220_{\angle 0^{\circ}} V & \bar{Z}_{R S}=366.4_{\angle 81.46^{\circ}} \Omega \\
\bar{V}_{S T}=220_{\angle-120^{\circ}} V & \bar{Z}_{R S}=325.07_{\angle 61.36^{\circ} \Omega} \\
\bar{V}_{T R}=220_{\angle-240^{\circ}} V & \bar{Z}_{R S}=361.37_{\angle-81.60^{\circ} \Omega}
\end{array}
$$

Phase current values and decomposition using Stokvis theorem are indicated in expression (16):

$$
\begin{array}{ll}
\bar{I}_{R S}=0.60_{\angle-81.46^{\circ} A} & \bar{I}_{R S d}=0.25_{\angle-49.36^{\circ} A} A \\
\bar{I}_{S T}=0.67_{\angle 178.64^{\circ} A} & \bar{I}_{R S i}=0.33_{\angle-23.21^{\circ} A} \\
\bar{I}_{T R}=0.60_{\angle-158.40^{\circ} A} & \bar{I}_{R S h}=0.46_{\angle-145.19^{\circ} A}
\end{array}
$$

The powers will be (5), (6):

$$
\begin{aligned}
& P_{u}=3 V_{R S d} \cdot I_{R S d} \cdot \cos \varphi_{d}=110.54 \mathrm{~W} \\
& Q_{u}=3 V_{R S d} \cdot I_{R S d} \cdot \operatorname{sen} \varphi_{d}=128.80 \mathrm{VAr} \\
& A_{u}=3 V_{R S d} \sqrt{\left(I_{R S i}^{2}+I_{R S h}^{2}\right)^{2}}=379.28 \mathrm{VAr} \\
& S_{u}=\sqrt{P_{u}^{2}+Q_{u}^{2}+A_{u}^{2}}=415.53 \mathrm{VA}
\end{aligned}
$$

Next we obtain the quantification of the energy phenomena from

the wave shapes of the instantaneous power components using the second procedure, by means of expressions (7), (9), (11), (13) and (14).

\section{A. Effective Power}

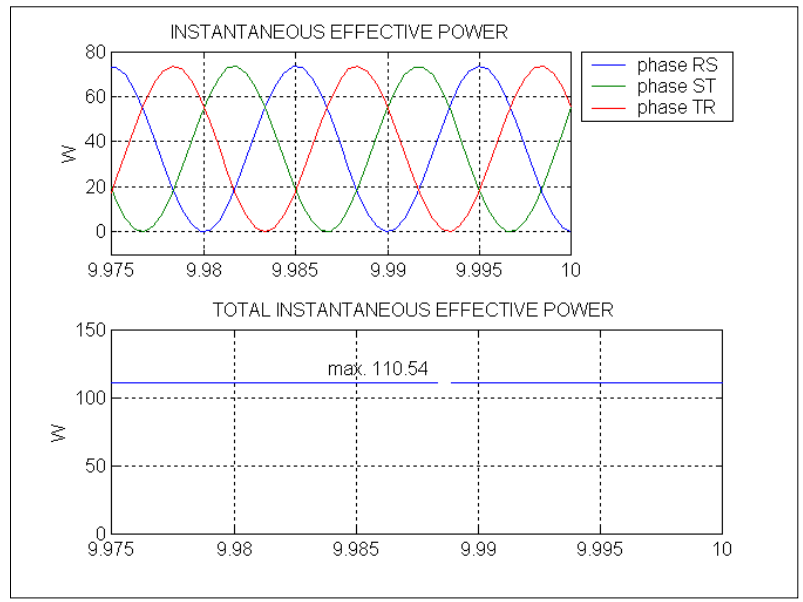

Fig. 6. Effective Power

$$
P_{u}=\sum_{j=R S, S T, T R} p_{j p}(t)=110.54 \mathrm{~W}
$$




\section{B. Dephase power}

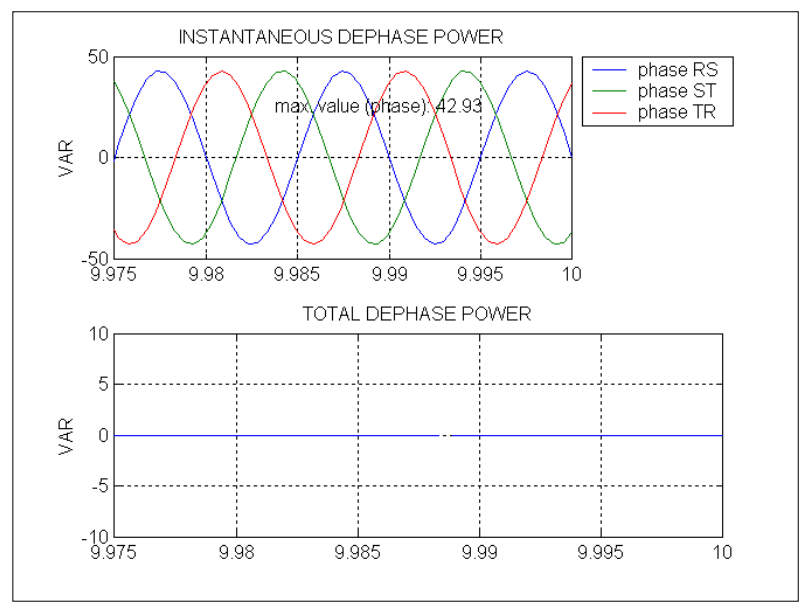

Fig. 7. Dephase Power

$Q_{u}=\sum_{j=R S, S T, T R} \max \left[p_{j q}(t)\right]=3 \cdot 42.93=128.79 \mathrm{VAr}$

\section{Asymmetry power associated with inverse current}

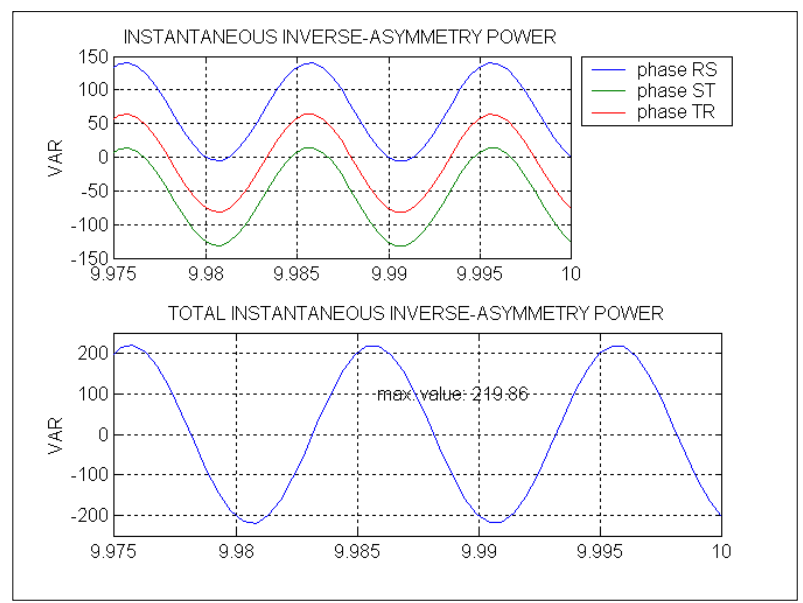

Fig. 8. Inverse-Asymmetry Power

$$
A_{\text {inverse }}=\sum_{j=R S, S T, T R} \max \left[p_{j A i}(t)\right]=219.86 V A r
$$

D. Asymmetry power associated with homopolar current

$$
\begin{aligned}
& A_{\text {homopolar }}=\sum_{j=R S, S T, T R} \max \left[p_{j A h}(t)\right]= \\
& =18.39+196.19+94.34=308.92 \mathrm{VAr}
\end{aligned}
$$

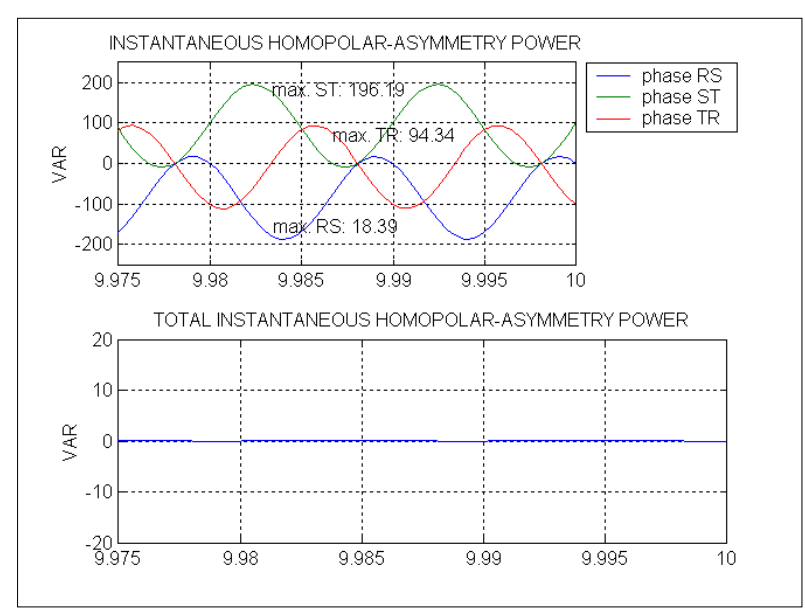

Fig. 9. Homopolar-Asymmetry Power

\section{E. Total asymmetry power}

From the values of expressions (12) and (13), and having into account that the asymmetry power can be obtained as the quadratic sum of its inverse and homopolar components (14):

$$
\begin{gathered}
A_{u}=\sqrt{A_{i}^{2}+A_{h}^{2}}=\sqrt{219.86^{2}+308.92^{2}}= \\
=379.27 \mathrm{VAr}
\end{gathered}
$$

This example illustrates that the quantification of the energy phenomena present in the power system can be obtained from the two procedures presented here, emphasizing the relationship between the temporal representations of the energy fluxes and the value of the power associated with the energy phenomenon.

\section{Conclusions}

The different energy phenomena occurring in power systems can be explained from the wave shapes of the instantaneous power components, quantifying such phenomena either from the magnitudes of the power system or from the wave shapes of the instantaneous power components of the system.

In load-unbalanced linear power systems there are three energy phenomena: useful energy transfer, dephase and asymmetry:

- $\quad$ Effective power is characterized by a senoid in each phase whose resultant is a one-directional power flux between generator and receiver.

- The dephase phenomenon manifests by three sinusoidal balanced fluxes, as opposed to what the classical theory states, according to which these power fluxes are unbalanced, as a consequence of including the effects of asymmetry. 
- The asymmetry phenomenon is characterized by three sinusoidal unbalanced fluxes, whose resultant is a senoid of nil mean value.

- It is possible to obtain the asymmetry power from the amplitude of the power fluxes typical of this energy phenomenon.

\section{Ackonowledgement}

We would like to thank the Foreign Language Coordination Office at the Polytechnic University of Valencia for their help in translating this paper.

\section{References}

[1] León Martínez, V.; Giner Garcia, J. "Método integral para la mejora de la eficiencia en las instalaciones eléctricas”. $5^{a}$ Jornadas Hispano-lusas de Ingeniería Eléctrica. July 1997. Salamanca.

[2] León Martínez, V., Montañana Romeu, J., Giner García, J., Cazorla Navarro, A., Sabater i Serra, R., Donderis Quiles, V. "Medida de la eficiencia y de la calidad en el suministro de los sistema eléctricos". Electrica'2000. International Conference. FIE 2000. Santiango de Cuba. July 2000.

[3] Sabater i Serra, R., Donderis Quiles, V., León Martínez, V. "Relación entre los fenómenos energéticos asociados a circuitos trifásicos lineales desequilibrados en cargas y sus formas de onda". $7^{a}$ Jornadas Hispano-lusas de Ingeniería Eléctrica. Madrid. Julio 2001. pp. 207-212.

[4] Montañana, J.; León, V.; Giner, J.; Cazorla, A. "Instrumento de medida de la eficiencia en el suministro de energía eléctrica en Baja Tensión".INTERCON 99, Lima 1999. 